\title{
Modeling dynamic fragmentation of concrete under high strain-rate loading
}

\author{
David Cereceda ${ }^{\mathrm{a}}$, Thomas Pavarini ${ }^{\mathrm{a}}$, Nitin Daphalapurkar ${ }^{\mathrm{a}, \mathrm{c}}$, Bryan Bewick $^{\mathrm{d}}$, \\ Lori Graham-Brady ${ }^{a, b, *}$ \\ ${ }^{a}$ Hopkins Extreme Materials Institute, Johns Hopkins University, Baltimore, MD 21218, USA \\ ${ }^{b}$ Department of Civil Engineering, Johns Hopkins University, Baltimore, MD 21218, USA \\ ${ }^{c}$ Department of Mechanical Engineering, Johns Hopkins University, Baltimore, MD 21218, USA \\ ${ }^{d}$ Protection Engineering Consultants, 14144 Trautwein Rd, Austin, TX, USA 78737
}

\begin{abstract}
Extreme high-rate loading conditions in structural materials trigger a complex process of fragmentation involving probabilistic, energetic and mechanical aspects. In this work we discuss a one-dimensional model based on [1] that captures the physics of dynamic fracture and fragmentation in concrete at strain rates from $10^{3}$ to $10^{5} / \mathrm{s}$, with particular interest in the higher strain rate values. In particular, the model considers a one-dimensional bar under a uniform tensile initial strain rate, with a stochastically varying strength. Initial results for the relationship between average fragment size and strain rate show good agreement with shock tube experiments on concrete panels. However, the predicted distribution of fragment size exhibits a smaller variance than that observed in the experiments. Future work will evaluate this difference in the results, which could be the result of the one-dimensionality of the model, heterogeneity of strain rate in the shock tube tests, experimental measurement errors, or a combination of all of these. Further investigations to extend the present model to other brittle materials like glass and concrete are also currently under development.
\end{abstract}

Keywords: concrete, dynamic fragmentation, high strain rates

\section{Introduction}

Understanding response of civilian structures to extreme environmental conditions is important for improved protection. Of importance, is understanding of the debris formation that results as a consequence of materials undergoing disintegration-also referred to as fragmentation. The highrate of loading (e.g. due to the blast wave front) can trigger fragmentation of materials with pieces much smaller than the size of a structural unit (e.g. panel, masonry block, slab) resulting in formation of debris. From a mechanics perspective, the first step is to gain understanding of the statistics of size and mass of fragments at the material level. Materials-level analysis can then inform the overall approach of debris formation from various type of civilian constructs for which

\footnotetext{
* corresponding author

Email addresses: david.cereceda@jhu.edu (David Cereceda), tpavari1@jhu.edu (Thomas Pavarini), nitin@jhu.edu (Nitin Daphalapurkar), bbewick@protection-consultants.com (Bryan Bewick), lori@jhu.edu (Lori Graham-Brady)
} 
the widely used material is concrete. This multiscale modeling approach, i.e. treating materialslevel and structures-level analysis separately, is a critical piece in the prediction of debris statistics from structures made of concrete.

Modeling dynamic brittle fragmentation at high strain rates is a complex process that involves probabilistic $[2,3]$, energetic $[4,5]$ and mechanical aspects $[1,6]$. Three different stages can be identified during this process: crack nucleation, crack propagation and crack coalescence. As it was first pointed out by Weibull [7], the largest defect plays a critical role in governing the onset of failure. At high strain rates, all the defects contribute to failure [8, 9]. These defects are inherent to the material, as a consequence of materials composition and processing conditions, among other things. Spatial distribution of defects contribute to the heterogeneity in material properties (e.g. strength) from point-to-point within the material domain. Then the heterogeneity of the material needs to be included in the model to capture crack nucleation [10]. Moreover, as a crack opens, it releases stress waves that propagate from the cracking point and protect neighboring areas from nucleating new cracks [11]. This mechanism of wave propagation was suggested by Mott [12] and it also needs to be incorporated in the model to characterize the second stage of the process.

The formulation of one-dimensional (1D) models that include these probabilistic, energetic and mechanical effects present some limitations to capture the geometric effects of crack propagation and subsequent fragment shapes. Two- and three-dimensional models can provide more accurate information but their physical and technical complexity are constraints that need to be evaluated as well. In this context, the development of one-, two- and three-dimensional results, based on the calibration between the 1D model and the experiments can be considered a valid approach.

The paper is organized as follows. First we briefly describe the experimental setup and analysis. We then provide detailed information about the simulation setup, a $1 \mathrm{D}$ model based on [1] that captures the physics of dynamic fracture and fragmentation in concrete at strain rates from $10^{3}$ to $10^{5} / \mathrm{s}$. In particular, the model considers a $1 \mathrm{D}$ bar under a uniform tensile initial strain rate, with a stochastically varying strength, and it includes the use of cohesive zone models to described the behavior of initiated cracks. In the Results section we report a parametric study to capture how sensitive are the fragment size and mass distributions to uncertainty in the material failure strength. Fragment size and mass distributions, and their comparison with experiments are also reported. We finish with a discussion of the results and the conclusions.

\section{Methodology}

\subsection{Experimental setup}

The objective of the experiments were to generate fragmentation data with an emphasis on collecting as much of the original sample mass as possible. $40.6 \mathrm{~cm}\left(16^{\prime \prime}\right)$ square plate samples, $5.08 \mathrm{~cm}(2$ ") thick, were placed at the end of the shock tube device supported by a plate with a 5.6 cm (14") circular opening. The configuration of experiments includes a catcher system that allows for physical collection of the fragments, and a high speed video (HSV) camera that is also used to collect information. Fragment-mass and velocity distributions were obtained using three different approaches: (i) from physical collection of fragments, (ii) videographic analysis of side-view HSV, and (iii) videographic analysis of rear-view HSV. The fragments were classified into size groups by using sieves of various sizes. Each size group was analyzed using the SigmaScan Pro software to assign a fragment count and the associated area for each individual fragment. Then, the overall mass of each sieve size was separated out to a mass for each individual fragment. The reader is referred to the work by [13] for a complete description of the experimental setup. 


\subsection{Simulation setup}

In this work, we are using a modified version of the 1D fragmentation model originally developed by Zhou et al.[1]. The governing equation of the model for a $1 \mathrm{D}$ bar oriented along the $x$-direction is the balance of momentum in one-dimension,

$$
\frac{\partial \sigma_{x}}{\partial x}=\rho \frac{\partial v}{\partial t}
$$

where $\rho$ is the density of the material, $\sigma$ is the stress and $v$ is the velocity. The one-dimensional bar of length $L$ is oriented along the $x$-axis with one of its ends fixed, and an uniform strain rate $\dot{\varepsilon}_{0}$ is also applied at the other end. Then, the initial and boundary conditions of the bar are

$$
\begin{gathered}
\sigma(x, 0)=\sigma_{0} ; \quad v(x, 0)=\dot{\varepsilon_{0}} \cdot x \\
v(0, t)=0 ; \quad v(L, t)=\dot{\varepsilon_{0}} \cdot L
\end{gathered}
$$

The heterogeneity in the material is introduced through the dependence of the critical strength $\sigma_{c}$ with the position along the bar, $\sigma_{c}=\sigma_{c}(x)$. In particular, $\sigma_{c}(x)$ is described by a Weibull distribution whose mean is given by the experimental value (Table 1). A new crack is nucleated at any point $x$ and time $t$ when the stress at that point and time exceeds this critical strength of the material, such as

$$
\sigma(x, t) \geqslant \sigma_{c}(x)
$$

Additionally, a linear cohesive zone model [14] is also included to incorporate the second stage of the fragmentation process, when cracks experience growing or closing behaviors after their nucleation. The one-dimensionallity of the problem guarantees that the entire set of equations can be solved using the method of characteristics described in detail by [1].

Table 1 shows the material properties from experiments [13], which contain information about the Young's modulus $E$, density $\rho$, critical strength $\sigma_{c}$ and fracture energy $G_{c}$ in concrete. However, some other parameters used in the material level model such as the standard deviation of strength are not known. In this work, we develop a parametric study of fragmentation to guarantee that the fragment size distributions predicted by the model capture the variability resulting from these unknown parameters. The question we seek to respond is: how sensitive is the fragment-size distribution to uncertainty in the material failure strength? We devise a parametric study that allows exploration of the variability in fragment-size distribution corresponding to variability in the failure strengths. We consider multiple realizations of spatial variability in order to obtain a representative result, independent of the spatial variability.

Table 1: Values of material properties in concrete from experiments [13].

\begin{tabular}{cccc}
\hline $\begin{array}{c}E \\
(\mathrm{MPa})\end{array}$ & $\begin{array}{c}\rho \\
\left(\mathrm{kg} / \mathrm{m}^{3}\right)\end{array}$ & $\begin{array}{c}\sigma_{c} \\
(\mathrm{MPa})\end{array}$ & $\begin{array}{c}G_{c} \\
(\mathrm{~N} / \mathrm{m})\end{array}$ \\
\hline 27580 & 2.323 & 3.59 & 75.30 \\
\hline
\end{tabular}




\section{Results}

\subsection{Fragment size distributions}

The parametric study described in the previous section is performed in concrete for a onedimensional bar of $5000 \mathrm{~mm}, 10^{6}$ nodes, strain rates from $10^{3}$ to $10^{5} / \mathrm{s}$, and a standard deviation of stress of 1,4 or $16 \%$. For each specific set of conditions, different tests are run to capture the random nature of the strength distribution with space. Finally, all the tests are collapsed into one single probability density function (pdf) that captures the variability in the data. Fig. 1 shows the pdf of fragment sizes for a standard deviation of stress of $1 \%$.

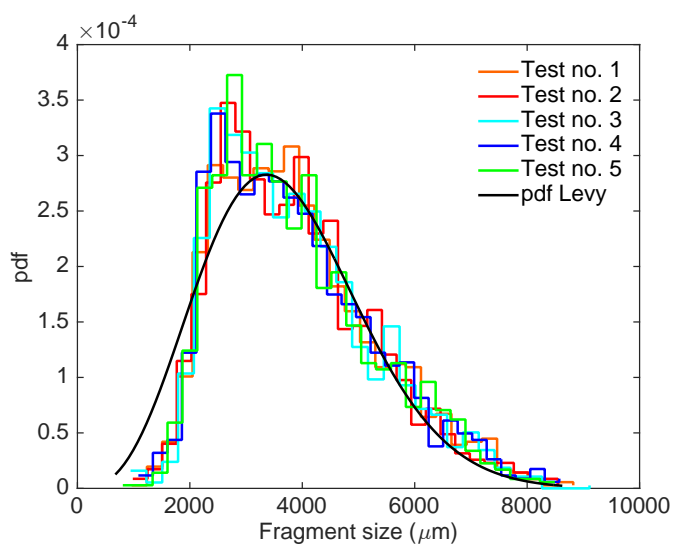

(a) $10^{3} \mathrm{~s}^{-1}$

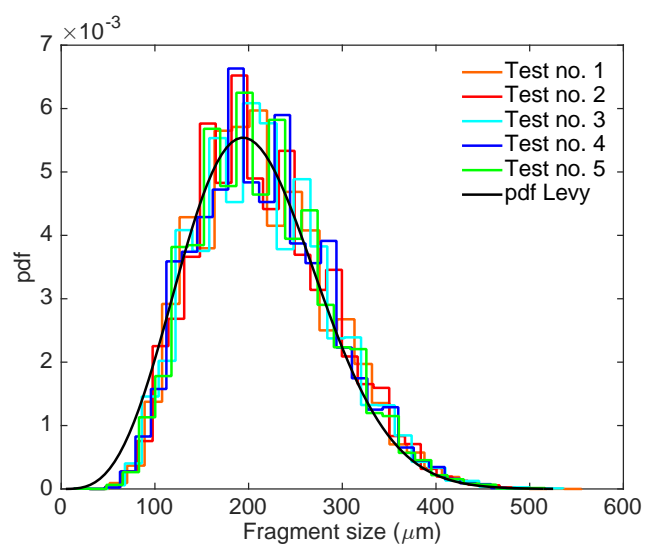

(c) $5 \cdot 10^{4} \mathrm{~s}^{-1}$

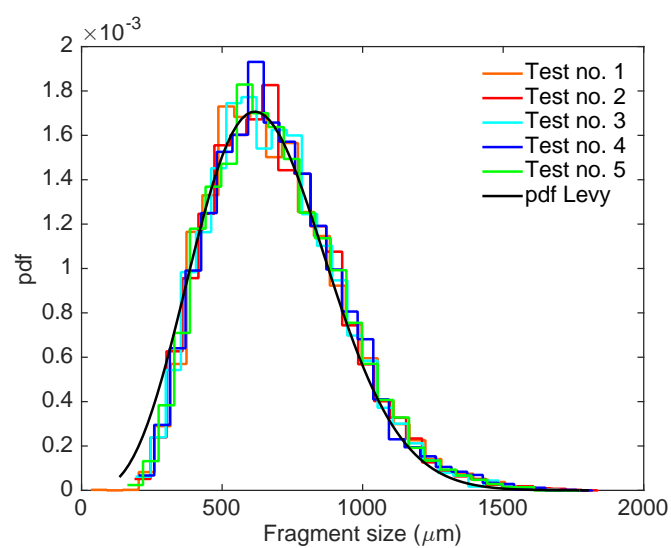

(b) $10^{4} \mathrm{~s}^{-1}$

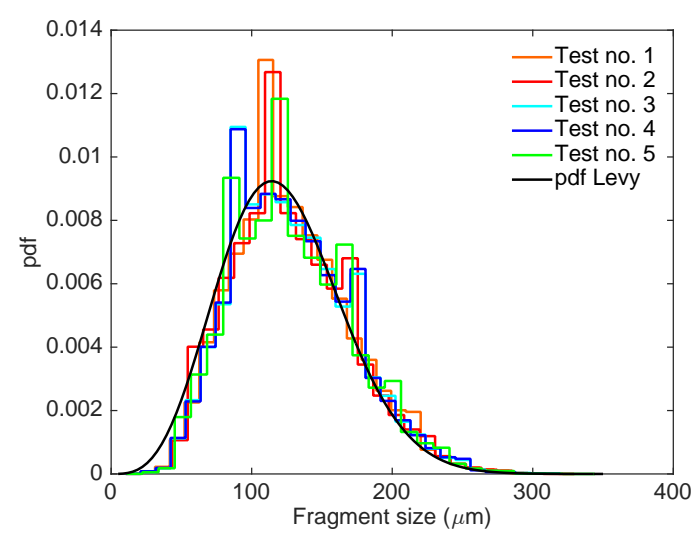

(d) $10^{5} \mathrm{~s}^{-1}$

Figure 1: Pdf of fragments-size distributions in concrete for a one-dimensional bar of $5000 \mathrm{~mm}, 10^{6}$ nodes, strain rates of $10^{3}, 10^{4}, 5 \cdot 10^{4}$ and $10^{5} \mathrm{~s}^{-1}$, and a standard deviation of stress of $4 \%$. For each strain rate, the entire family of tests is collapsed into one single pdf using the distribution suggested by Levy [15].

A comprehensive revision of the state-of-the-art on types of fragment size distributions was presented in [16]. Based on the Bayesian Information Criterion ${ }^{1}$, we select the generalized gamma

\footnotetext{
${ }^{1}$ The Bayesian Information Criterion (BIC) is a criterion based on the likelihood function to select, among a finite set of models, the most accurate model to fit a specific set of data [17].
} 
distribution suggested by Levy [15] to fit our fragmentation data. The cumulative distribution function for fragment size, using this type of distribution is given by

$$
\begin{gathered}
F_{D}(d)=P[D \leqslant d] \\
F_{D}(d)=\left(1+\left(\frac{d}{\mu}\right)^{\beta}\right) \cdot \exp \left(-\left(\frac{d}{\mu}\right)^{\beta}\right)
\end{gathered}
$$

where $P[D \leqslant d]$ is the probability of finding a size of largest length $d$ greater than a specific length $D, \mu$ is a scale parameter and $\beta$ is a shape parameter.

Table 2 shows the values of the fitting parameters of fragment size distributions for the entire strain rate regime and different values of stress deviation.

Table 2: Fitting parameters $\mu$ and $\beta$ of the Levy distribution [15] in concrete for a one-dimensional bar of $5000 \mathrm{~mm}$, $10^{6}$ nodes, strain rates of $10^{3}, 10^{4}, 5 \cdot 10^{4}$ and $10^{5} \mathrm{~s}^{-1}$, with a standard deviation of stress of 1,4 and $16 \%$.

\begin{tabular}{ccccc} 
& \multicolumn{4}{c}{ Strain rate $\left(\mathrm{s}^{-1}\right)$} \\
\hline Stress deviation (\%) & $10^{3}$ & $10^{4}$ & $5 \cdot 10^{4}$ & $10^{5}$ \\
\hline \multirow{2}{*}{1} & $\mu=2943.9$ & $\mu=496.49$ & $\mu=157.47$ & $\mu=91.84$ \\
& $\beta=2.0$ & $\beta=2.13$ & $\beta=2.09$ & $\beta=2.04$ \\
4 & $\mu=2741.3$ & $\mu=504.23$ & $\mu=158.84$ & $\mu=93.44$ \\
& $\beta=1.9$ & $\beta=2.08$ & $\beta=2.12$ & $\beta=2.09$ \\
\multirow{2}{*}{16} & $\mu=2252$ & $\mu=521.68$ & $\mu=159.49$ & $\mu=94.02$ \\
& $\beta=1.8$ & $\beta=1.89$ & $\beta=2.12$ & $\beta=2.12$ \\
\hline
\end{tabular}

\subsection{Fragment mass distributions}

The cumulative distribution function for fragment mass distribution, assuming a different set of fitting parameters from those used in Sect. 3.1, is given by

$$
\begin{gathered}
F_{M}(m)=P[M \leqslant m] \\
F_{M}(m)=\left(1+\left(\frac{m}{\mu^{\prime}}\right)^{\beta^{\prime}}\right) \cdot \exp \left(-\left(\frac{m}{\mu^{\prime}}\right)^{\beta^{\prime}}\right)
\end{gathered}
$$

where $P[M \leqslant m]$ is the probability of finding a mass of largest weight $m$ greater than a specific mass $D$.

The relation between probability of mass and size distribution is

$$
F_{M}(m)=P\left[k \rho D^{3} \leqslant m\right]=P\left[D \leqslant\left(\frac{m}{k \rho}\right)^{\frac{1}{3}}\right]=F_{D}\left(\left(\frac{m}{k \rho}\right)^{\frac{1}{3}}\right)
$$

Then the cumulative fragment function distribution of mass that corresponds to the cumulative fragment distribution of size from eq. 6 can be rewritten as 


$$
F_{M}(m)=\left(1+\left(\frac{m}{\rho k \mu^{3}}\right)^{\frac{\beta}{3}}\right) \cdot \exp \left(-\left(\frac{m}{\rho k \mu^{3}}\right)^{\frac{\beta}{3}}\right)
$$

Thus, the same type of distribution can be used to fit both, size and mass, statistics. If the pdf statistics of size distribution $(\beta, \mu)$ are known, then one can find the statistics of mass distribution using $\beta^{\prime}=\frac{\beta}{3}$ and $\mu^{\prime}=\rho k \mu^{3}$, where $k$ is a shape factor.

Following the same idea that it was described in the previous section, we present a parametric study of fragment mass distributions to guarantee that: (i) the fragment mass distributions predicted by the model capture the variability resulting from these unknown parameters and (ii) the random nature of the strength distribution with space is also captured. Fig. 2 shows the pdf of fragment mass distributions for a standard deviation of stress of $1 \%$. Table 3 shows the values of the fitting parameters of fragment mass distributions for the entire strain rate regime and different values of stress deviation.

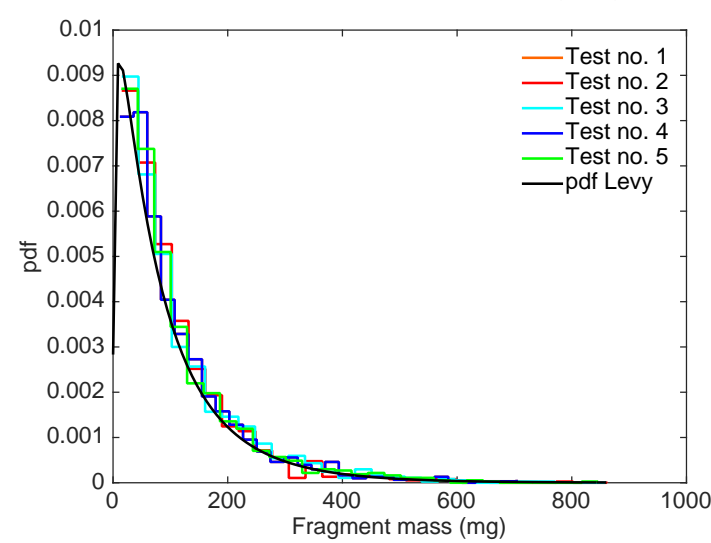

(a) $10^{3} \mathrm{~s}^{-1}$

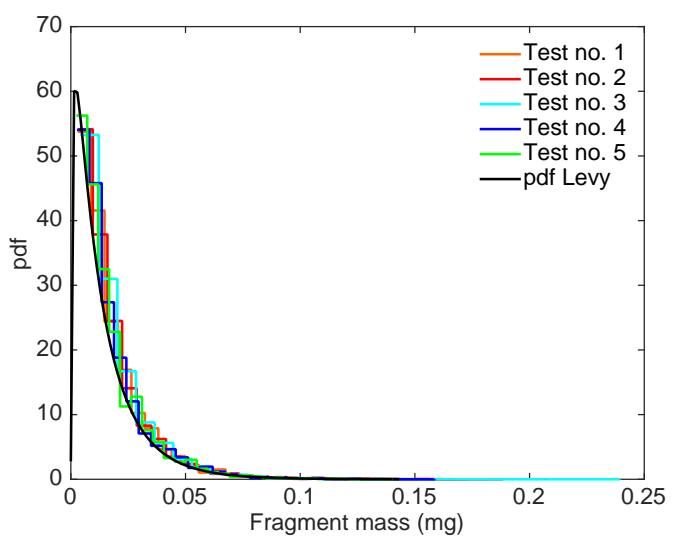

(c) $5 \cdot 10^{4} \mathrm{~s}^{-1}$

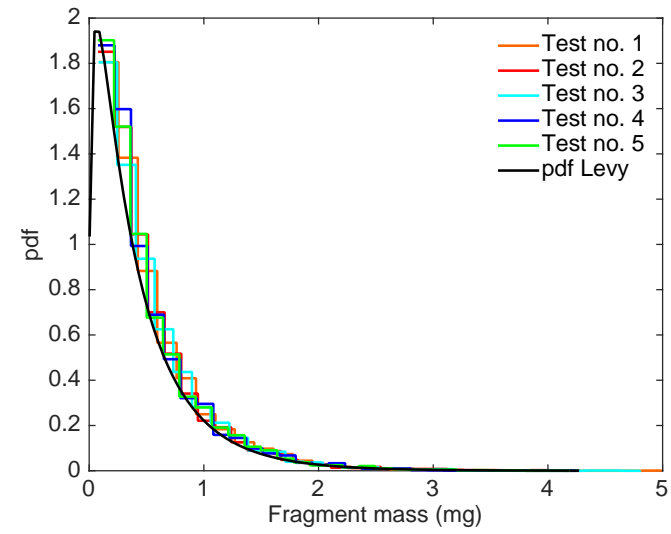

(b) $10^{4} \mathrm{~s}^{-1}$

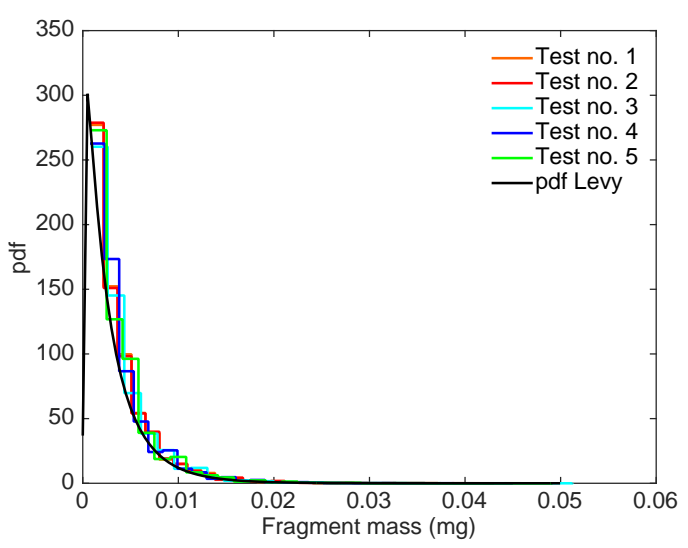

(d) $10^{5} \mathrm{~s}^{-1}$

Figure 2: Pdf of fragments-mass distributions in concrete for a one-dimensional bar of $5000 \mathrm{~mm}, 10^{6}$ nodes, strain rates of $10^{3}, 10^{4}, 5 \cdot 10^{4}$ and $10^{5} \mathrm{~s}^{-1}$, and a standard deviation of stress of $1 \%$. For each strain rate, the entire family of tests is collapsed into one single pdf using the distribution suggested by Levy [15]. 
Table 3: Fitting parameters $\mu^{\prime}$ and $\beta^{\prime}$ of the Levy distribution [15] in concrete for a one-dimensional bar of 5000 $\mathrm{mm}, 10^{6}$ nodes, strain rates of $10^{3}, 10^{4}, 5 \cdot 10^{4}$ and $10^{5} \mathrm{~s}^{-1}$, with a standard deviation of stress of 1,4 and $16 \%$.

\begin{tabular}{ccccc}
\cline { 2 - 5 } & \multicolumn{4}{c}{ Strain rate $\left(\mathrm{s}^{-1}\right)$} \\
\hline Stress deviation (\%) & $10^{3}$ & $10^{4}$ & $5 \cdot 10^{4}$ & $10^{5}$ \\
\hline \multirow{2}{*}{1} & $\mu^{\prime}=30.645$ & $\mu^{\prime}=0.146$ & $\mu^{\prime}=4.7 \mathrm{e}-3$ & $\mu^{\prime}=9.375 \mathrm{e}-4$ \\
& $\beta^{\prime}=0.67$ & $\beta^{\prime}=.698$ & $\beta^{\prime}=0.692$ & $\beta^{\prime}=0.67$ \\
4 & $\mu^{\prime}=23.276$ & $\mu^{\prime}=0.151$ & $\mu^{\prime}=4.813 \mathrm{e}-3$ & $\mu^{\prime}=9.75 \mathrm{e}-4$ \\
& $\beta^{\prime}=0.601$ & $\beta^{\prime}=0.675$ & $\beta^{\prime}=0.699$ & $\beta^{\prime}=0.686$ \\
\multirow{2}{*}{16} & $\mu^{\prime}=20.086$ & $\mu^{\prime}=0.163$ & $\mu^{\prime}=4.75 \mathrm{e}-3$ & $\mu^{\prime}=1 \mathrm{e}-3$ \\
& $\beta^{\prime}=0.583$ & $\beta^{\prime}=0.605$ & $\beta^{\prime}=0.689$ & $\beta^{\prime}=0.696$ \\
\hline
\end{tabular}

\subsection{Comparison with experiments}

Prior to the formulation of calibration laws between the 1D model and experiments, some more understanding about the comparison between experimental and simulation results is needed. In this direction, Fig. 3 shows the log-log curve of fragment mass vs. strain rate when comparing experiments [13] and 1D fragmentation simulations in concrete. The pdf of normalized fragment size from experiments and simulations are also plotted in Fig. 4 to quantify how accurate are the shape and scatter of the fragment distributions when compared to each other.

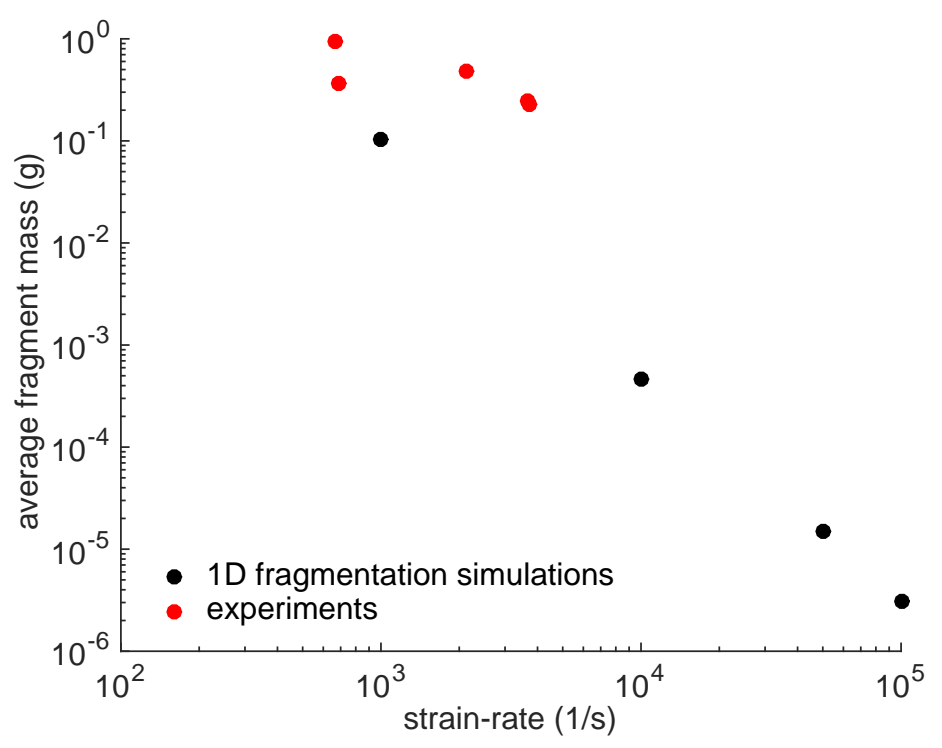

Figure 3: Average fragment mass vs strain rates in concrete.

\section{Discussion and conclusions}

In Sections 3.1 and 3.2 we have provided information about the fragment-size and fragmentmass distributions in a one-dimensional bar of concrete at strain rates from $10^{3}$ to $10^{5} \mathrm{~s}^{-1}$. The 


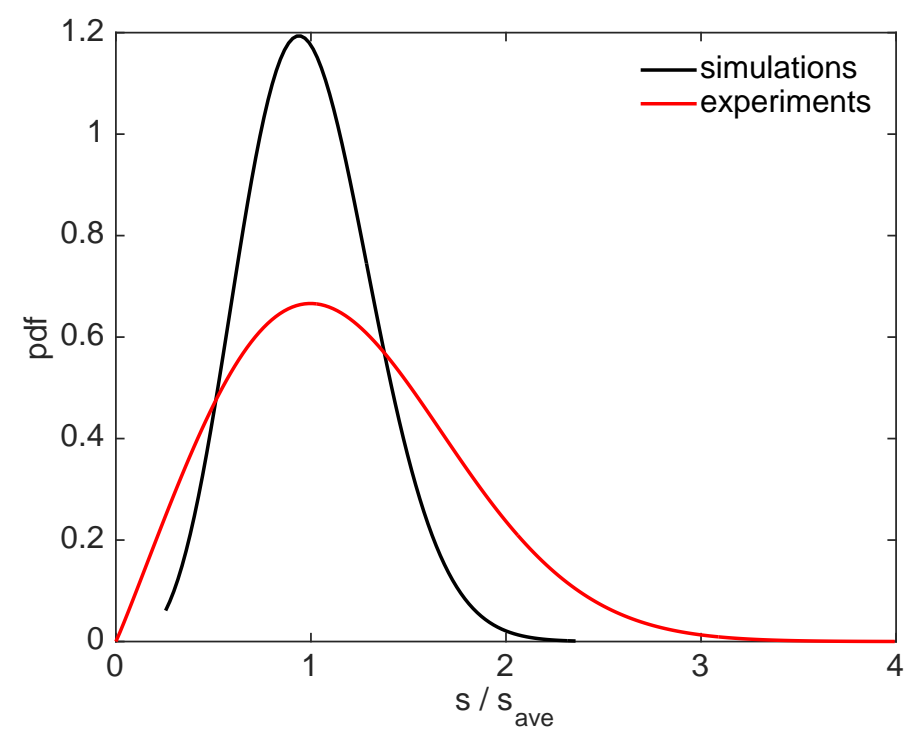

Figure 4: Comparison of normalized fragment-size pdf from 1D simulations with 3D shock tube experiments in concrete.

parametric study was designed to explore the variability in the distributions with the uncertainty in the material failure strength, and it examines two factors: (i) first, for each specific set of strain rate and stress deviation conditions, five different tests are run to capture the random nature of strength distribution along the bar; (ii) second, a stress deviation range from 1 to $16 \%$ was studied to cover the unknown values of stress deviation in the experiments [13]. The fitting parameters for fragment-size $(\mu, \beta)$ and fragment-mass $\left(\mu^{\prime}, \beta^{\prime}\right)$ shown in Tables 2 and 3 reveal that $\mu$ and $\mu^{\prime}$ decrease when increasing strain rate, while $\beta$ and $\beta^{\prime}$ remain constant for the entire strain-rate regime.

The fragment mass vs. strain rate results presented in Fig. 3 show a decrease of fragment mass with strain rate both for experiments and 1D fragmentation simulations. This is in agreement with previous works in the field $[1,6,18]$ and the well-accepted physical principle of smaller fragmentsizes (or fragment-mass) associated with higher strain rates. Regarding the comparison between experiments and 1D fragmentation simulations, the order of magnitude of the error is less than one, but some more experimental data at higher strain rates and values of the uncertainty in experimental measurements are both required before formulating any further conclusion.

The shape of the normalized fragment size distributions shown in Fig. 4 reveal a reasonable match between experiments and simulations, but the scatter is underestimated by simulations. These differences could be the result of the one-dimensionality of the model, heterogeneity of strain rate in the shock tube tests, experimental measurement errors, or a combination of all of these. As it was presented by [15], the probability density functions at different strain rates collapse into the same one when the fragment size is normalized, then the previous comparison between experimental and simulation pdf can be extended to the entire range of strain rate.

\section{Acknowledgements}

All simulations were run on Maryland Advanced Research Computing Center (MARCC). 


\section{References}

[1] F. Zhou, J.-F. Molinari, K. Ramesh, A cohesive model based fragmentation analysis: effects of strain rate and initial defects distribution, International journal of solids and structures 42 (18) (2005) 5181-5207.

[2] L. Oddershede, P. Dimon, J. Bohr, Self-organized criticality in fragmenting, Physical review letters 71 (19) (1993) 3107.

[3] P. Elek, S. Jaramaz, Fragment size distribution in dynamic fragmentation: Geometric probability approach, FME Transactions 36 (2) (2008) 59-65.

[4] D. Grady, Local inertial effects in dynamic fragmentation, Journal of Applied Physics 53 (1) (1982) 322-325.

[5] L. Glenn, A. Chudnovsky, Strain-energy effects on dynamic fragmentation, Journal of applied physics 59 (4) (1986) 1379-1380.

[6] F. Zhou, J.-F. Molinari, K. Ramesh, Analysis of the brittle fragmentation of an expanding ring, Computational Materials Science 37 (1) (2006) 74-85.

[7] W. Weibull, A statistical theory of the strength of materials, no. 151, Generalstabens litografiska anstalts förlag, 1939.

[8] N. P. Daphalapurkar, K. Ramesh, L. Graham-Brady, J.-F. Molinari, Predicting variability in the dynamic failure strength of brittle materials considering pre-existing flaws, Journal of the Mechanics and Physics of Solids 59 (2) (2011) 297-319.

[9] G. Hu, J. Liu, L. Graham-Brady, K. Ramesh, A 3d mechanistic model for brittle materials containing evolving flaw distributions under dynamic multiaxial loading, Journal of the Mechanics and Physics of Solids 78 (2015) 269-297.

[10] F. Zhou, J.-F. Molinari, Stochastic fracture of ceramics under dynamic tensile loading, International journal of solids and structures 41 (22) (2004) 6573-6596.

[11] S. Levy, J.-F. Molinari, Dynamic fragmentation of ceramics, signature of defects and scaling of fragment sizes, Journal of the Mechanics and Physics of Solids 58 (1) (2010) 12-26.

[12] N. Mott, Fragmentation of shell cases, in: Proceedings of the Royal Society of London A: Mathematical, Physical and Engineering Sciences, Vol. 189, The Royal Society, 1947, pp. 300-308.

[13] B. Bewick, G. Rolater, M. Sanai, A. Ziemba, Debris hazards due to overloaded conventional construction facades.

[14] G. T. Camacho, M. Ortiz, Computational modelling of impact damage in brittle materials, International Journal of solids and structures 33 (20) (1996) 2899-2938.

[15] S. Levy, Exploring the physics behind dynamic fragmentation through parallel simulations, Ph.D. thesis (2010).

[16] C. B. Laney, Universal size distributions for liquid and solid fragmentation, submitted to Physical Review E.

[17] G. Schwarz, Estimating the dimension of a model, The annals of statistics 6 (2) (1978) 461-464.

[18] F. Zhou, J.-F. Molinari, K. Ramesh, Effects of material properties on the fragmentation of brittle materials, International journal of fracture 139 (2) (2006) 169-196. 